\title{
Nachruf Prof. Dr. med. Friedrich Trendelenburg
}

\author{
Gerhard W. Sybrecht
}

\author{
In Memoriam Prof. Dr. med. Friedrich Trendelenburg
}

Der Name Trendelenburg gehört zu den berühmten Eigennamen, die den Ärzten geläufig sind. Friedrich Trendelenburg war sich dem damit verbundenen Anspruch an seine Person und an sein Handeln bewusst und erhob ihn zu einer seiner Lebensmaximen. Mit seinem Familiennamen verbindet sich die Trendelenburg-Operation, eine heroische Maßnahme zur Lebensrettung bei fulminanter pulmonaler Embolie, die sein Großvater Friedrich (1844-1924) erstmals 1920 durchführte. Bekannt ist auch die Trendelenburg-Lagerung (Becken-Bein-Hochlagerung beim Schock).

Neben dem Chirurgen Friedrich Trendelenburg, der in Bonn, Leipzig und Rostock wirkte und bereits 1869 mit ChloroformNarkose mit selbst entwickelten Tracheotomie-Tubus-Sets (bestehend aus Tubus mit Blockmanschette und Narkosetrichter) experimentierte, ist der Urgroßvater Friedrich Adolf Trendelenburg als protestantischer spätidealistisch-neukantianischer Philosoph und neuhumanistischer Bildungstheoretiker in der deutschen Wissenschaftsgeschichte berühmt.

Friedrich Trendelenburg kam am 26. August 1916 als Sohn des Ministerialdirektors Dr. jur. hc. Friedrich Trendelenburg und seiner Frau Margret, geborene Schwartz, in Berlin zur Welt. Friedrich Trendelenburg fühlte sich stets dem Ganzen verpflichtet und übernahm bereitwilligst Verantwortung im überregionalen Bereich.

Nach dem Abitur am Humanistischen Schiller-Gymnasium in Berlin studierte er Medizin in Lausanne, Königsberg, München und Berlin und absolvierte eine Weiterbildung in Berlin, bevor ihn sein Beruf als Arzt und seine eigene Erkrankung an Tuberkulose nach Davos in die Nachbarschaft des „Zauberbergs“ führte.

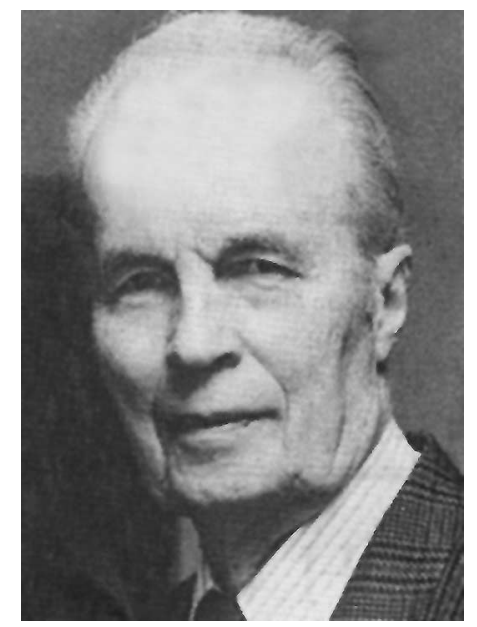

Universitätsprofessor Dr. med. J. Chr. Friedrich Trendelenburg, Wegbereiter der Pneumologie von der Phtisiologie in die Innere Medizin, am 26. 8. 1916 in Berlin geboren und am 7. 9. 2004 an den Folgen einer Pneumonie in Basel verstorben.

Als Oberarzt und wenig später als Chefarzt des Sanatorium Wolfgang in Davos war er maßgeblich an der Neuausrichtung dieser deutschen Stiftung für vorwiegend mittellose, lungenkranke deutsche Patienten in einer Asthmaklinik verantwortlich. Sie wurde weltberühmt und vor allem dadurch erfolgreich, dass Hausstaubmilben als Allergenauslöser in dieser Höhe nicht anzutreffen sind. Später wurde der Sanatoriumsanteil dieser Institution ganz geschlossen. Die Ära der Phtisiologie in dieser Institution, weit weniger mondän als in Thomas Mann's Zauberberg, war zu Ende.

1964 wechselte Trendelenburg von Davos-Wolfgang auf die Stelle eines leitenden Arztes der Abteilung für Pneumonologie der I. Medizinischen Klinik der Universität des Saarlandes in Homburg, deren Direktor Doenike L. war, und habilitierte sich für das Fach 
Innere Medizin, insbesondere Lungenkrankheiten. Das Thema seiner Habilitationsschrift „antibakterielle Chemotherapie der Tuberkulose“ und die Überschrift „Aus dem Sanatorium Wolfgang, der Stiftung Deutscher Heilstätten Davos und Agra“ (Chefarzt Dr. F. Trendelenburg) zeigt, dass die medizinische Fakultät des Saarlandes die Leistungen Trendelenburg's auf dem Gebiet der modernen Therapie der Tuberkulose mit der damals bekannten und sich gerade entwickelnden Chemotherapeutika honorierte. Diese junge Fakultät, die auf eine französische Initiative der Fakultät in Nancy 1947 zurückgeht und im Gelände der „Anstalt“ in Homburg/Saar entstand, gab Trendelenburg die Chance, die Robert Koch-Klinik, die über Jahrzehnte auch als Sanatorium bekannt war, zu einer neuen modernen Lungenklinik umzubauen. Trendelenburg wurde zu einem erfolgreichen Verfechter der Erkenntnis, dass Innere Medizin ohne die Pneumologie unvollständig ist und er überzeugte die Medizinische Fakultät davon, dass aus der ehemals nicht untergliederten Medizinischen Klinik eine solche mit 5 Abteilungen wurde. 1969 wurde er zum Direktor der Abteilung für Pneumonologie (Trendelenburg legte Wert auf diese nicht mehr gebräuchliche Bezeichnung, weil er die Kombination von Latein-Griechisch in Pneumologie als inkorrekt empfand) und führte mit seinen 4 Co-Direktoren (Abt. f. Hämatologie und Gastroenterologie, Kardiologie, Nephrologie und Poliklinik) die Medizinische Klinik der Universitätsklinik des Saarlandes. Es gelang ihm aus der Robert-Koch-Klinik die erste in Deutschland selbständige universitäre pneumologische Klinik zu machen. Er war sehr stolz darauf, die Trennung infektiöser (tuberkulöser Patienten) von den anderen internistischen erkrankten Lungenpatienten aufzuheben und diese unter das Dach der Inneren Medizin zurückzubringen.

Bei Übernahme der Klinik fand er 86 Betten mit einer Belegung von etwa $60 \%$ Tuberkulosekranken vor, bei seiner Emeritierung 1985 und meiner Übernahme waren es nur noch 5-10\% der Patienten, die mit Mykobakterien infiziert waren. In nur 20 Jahren ärztlicher Tätigkeit hatte sich das Spektrum der Aufgaben so stark verändert, dass er selbst von der neuen Epidemie infolge inhalativer Noxen nach dem Abklingen der Epidemie der Tuberkulose sprach.

Der schon zitierte Charakterzug, Verantwortung zu übernehmen, wann immer sie erforderlich ist, zeigte sich nicht nur in den bisher skizzierten Aktivitäten, sondern lässt sich auch an weiteren weitsichtigen Entwürfen dokumentieren, von denen die deutsche Pneumologie profitierte. 1963 gründete er die Wissenschaftliche Arbeitsgemeinschaft für Therapie von Lungenerkrankungen (W.A.T.L.) und war bis $1994 \mathrm{ihr}$ erster Vorsitzender. Dies geschah im Vorgriff auf die jetzt so modern gewordenen Netzwerke im Zusammenschluss zur Durchführung multizentrisch kontrollierter Therapiestudien.

In dem Bemühen, die hier gewonnenen Ergebnisse und praktischen Implikationen auch zu veröffentlichen, war es nur konsequent, dass er sich als Co-Editor der internationalen Fachzeitschrift LUNG engagierte, die aus Brauers Beiträgen im SpringerVerlag entstand. Später wurde er Consultant Editor des European Journal of Respiratory Diseases, dem Vorläufer des heutigen European Respiratory Journal (ERJ). Im Bereich der Phtisiologie fand Trendelenburg Strukturen vor, die bereits im 19. Jahrhundert gelegt wurden und in denen er sich auch wohl fühlte, z.B. als Präsident des Deutschen Zentralkomitees zur Bekämpfung der Tuberkulose. Dieses Amt hatte er 1985 und 1986 inne. Er erkannte aber auch, dass alte Strukturen sich anpassen müssen an die neuen Gegebenheiten und so war er Gründungsmitglied der Paul-Ehrlich-Gesellschaft für Chemotherapie und Gründungsmitglied der Societas Europaea Pathophysiologiae Clinicae Respiratoriae (SEPCR) und später dann auch 1984 Gründungsmitglied der Societas Europaea Pneumologiae, den beiden Vorläufern der European Respiratory Society, die 1990 gegründet wurde. Selbstverständlich leistete er als Exponent der internistisch verstandenen Pneumologie einen großen Beitrag für die Innere Medizin, indem er maßgeblich an der Gründung des Berufsverbandes Deutscher Internisten beteiligt war und als Sprecher der Teilgebiete der Inneren Medizin wirkte. Seine Bemühungen um Qualität innerhalb der Inneren Medizin, insbesondere in der Ausbildung, zeigte sich an der Erstellung des Weiterbildungskataloges für Internisten und auch der Internisten mit Schwerpunkten. Aus der Sicht der betroffenen Patienten war es wichtig, dass er sowohl das Saarländische Tumorzentrum und überwiegend die Deutsche Atemwegsliga mitgründete.

Die Übernahme dieser großen Verantwortlichkeiten wurde ihm gedankt und honoriert. So erhielt er 1987 vom Berufsverband Deutscher Internisten die Günther-Budelmann-Medaille und er wurde Ehrenmitglied der Süddeutschen Gesellschaft für Pneumologie und der Deutschen Gesellschaft für Pneumologie, für die er 1986 als Präsident des Jahreskongress in Saarbrücken nach seiner Emeritierung veranstaltete. Übernahme von Verantwortung und Gestaltung von Strukturen waren wesentliche Charakterzüge von Trendelenburg. Ein weiterer, ebenfalls aus der Familientradition begünstigter, war derjenige der Erziehung zur Wissenschaftlichkeit. Zu seinen Maximen ärztlicher und klinischer Tätigkeit notierte er: „Erst 2000 Jahre wissenschaftlichen Arbeitens führten zu der allerdings immer noch lückenhaften (siehe Homöopathie, Akupunktur, Naturheilkunde und anderes mehr) Erkenntnis, welchen Respekt wir den Tatsachen zu schenken haben und wie konzentriert und selbstkritisch die Beobachtungen durchgeführt werden müssen“. Er verspürte, „wie in dem von ihm vertretenen Bereich der Pneumologie am Beispiel der großen Volkskrankheiten Tuberkulose, Asthma, Bronchialkarzinom nach nur über 1000 Jahren belegt ist, dass autistisch undiszipliniertes Denken (Bläuler) oft nur simple Arroganz zu Irrtümern oder verhängnisvollen Fehlentwicklungen zum Schaden von Millionen von Patienten in den vergangenen Jahrhunderten geführt hat". Er erinnerte in diesem Kontext an die frühere Erkenntnis, „dass die der Tuberkulose so nahe verwandte Lepra eine übertragbare Krankheit ist, womit der oft rigorose Weg ihrer Bekämpfung durch Isolierung vorgezeichnet war“.

Trendelenburg empfand Dankbarkeit dafür, dass er wertvolle Erkenntnisse aus dem Studium der Wissenschaftstheorie (Erkenntnistheorie, kognitive Wissenschaften) erlangte, deren Ausgangspunkt für ihn die humanistische Schulbildung insbesondere mit denen das abendländliche Denken so bestimmenden Lehren von Sokrates und Platon war. Er folgerte daraus, „dass nur ständiges kritisches Überprüfen des Handelns in der Medizin den Fortschritt gewährleistete. Ungeordnetes, irrationales Denken und metaphysisches Spekulieren ist kaum weniger verbreitet als im Mittelalter, nur viel besser kaschiert durch moderne PR-Methoden“. 
Ich habe diese Zitate seinen Aufzeichnungen entnommen, die er als „Epikrise meiner klinischen und universitären Tätigkeit“ hinterlassen hat, und empfinde sie als Memento eines großen vielseitigen Mannes, vor dem sich die deutschen Pneumologen verneigen: „Bene meritus est“.
Friedrich Trendelenburg verstarb am 7. September 2004. Er hinterlässt 3 Söhne: Dr. Christian Trendelenburg, Toxikologe in Basel, Dr. George Trendelenburg, Arzt an der Charité in Berlin und Dr. Marten Trendelenburg, Oberarzt am Universitätsspital in Basel sowie deren Familien mit insgesamt 6 Enkelkindern.

\section{Buchbesprechung}

\section{Gravity and the Lung, Lessons from Microgravity}

Prisk GK, Paiva M, West JB (eds)

408 S. Marcel Dekker, New York. \$175

ISBN 0-8247-0570-X

Der physikalisch pneumologisch geschulte Zeitgenosse liest den 160. Band in der Marcel-Dekker-Reihe„Lung Biology in Health and Disease" herausgegeben von Claude Lenfant, über die glänzend geschriebene moderne Eroberungsgeschichte des Weltund Luftraums sowie den Wassertiefen (siehe auch Band 132, „The lung at depthe“, the undersea world!) unseres Planeten mit besonderem Vergnügen. Die einzelnen Kapitel umfassen Beschleunigungseffekte auf die Lungen- und Thoraxmechanik, die Ventilations- und Perfusionsverteilung sowie den Aerosoltransport in den Atemwegen. Die Änderungen des Gasaustausches unter verschiedenen Körperpositionen in Ruhe als auch bei unterschiedlichen ergometrischen Belastungen (isometrisch und dynamisch) finden ebenso profunde Erwähnung wie die kardiorespiratorische Kreislaufadaptation sowie die interstitielle Flüssigkeitsbilanz bei Einwirkung von Schwerkraftänderungen auf das thorakopulmonale System und das pleurale Kompartiment. Die Atemregulation wird unter normo-, hypo- und hyperbaren Sauerstoffatmosphären sowie Kohlendioxydpartialdrucken entsprechend den Bedingungen wie sie in U-Booten und Weltraumstationen vorkommen, an Hand von Kurz- und Langzeitexperimenten diskutiert. Dekompressionskrankheiten wie sie bei Arbeiten außerhalb von Raumschiffstationen in Druckanzügen geschehen können, werden den Dekompressionsunfällen nach Tauchgängen im Wasser gegenübergestellt und in aeronautischen Laboratorien eingehend analysiert. Die hyperbaren Therapieschemata mit ihren Gefahren der Kurz- und Langzeitsauerstofftoxizität auf Lunge und Zentralnervensystem finden besondere Beachtung.
Das Buch ist eine physiologische Basis für jeden Marine-Taucherarzt sowie Flug- und Weltraummediziner. Es fasst vorwiegend die Erfahrungen der Nasa und der US-Navy zusammen. Es kommen zwar auch zwei Italienische, ein Belgischer sowie Schwedischer Forscher als Kapitelautoren zu Wort, das Europäische Raumfahrtsprogramm findet aber im Gegensatz zum Russischen kaum Erwähnung. Man fragt sich, wieso es nicht möglich war, auch ehemalige Sowjetrussische Forscher, welche uns lange Zeit sowohl was Raketentechnik und Raumfahrt betrifft überlegen waren (Erste Weltraumfahrt des Hundes Laika und des Cosmonauten Gagarin 12. 4. 61, MIR 1986 mit Weltrekorden von Daueraufenthalten in Weltraumstationen), in einem Kapitel zu Wort kommen zu lassen.

Die Erkenntnis, dass die Schwerkraftwirkung mit relativer Hyperoxie und Hyperventilation das Angehen der Tuberkulose (Tb) im Lungenapex zumindest bei aufrechtem Thorax begünstigt, verdanken wir deutschen Pionieren des 19. Jahrhunderts. Die Höhenliegekur war die einzige physikalisch-therapeutische Maßnahme, die uns gegen die Krankheitsprogredienz der Tb bis zur ersten evidenzbasierten Tuberkulostaticatherapiestudie in England 1952 zur Verfügung stand. Zur gleichen Zeit erlaubte die Einführung der Radionukleide die regionale Lungenfunktion unter veränderten Schwerkraftsbedingungen eingehend zu studieren, was neue diagnostische aber leider nur wenige therapeutische Optionen bisher geschaffen hat. Die Forschung im schwerelosen und beschleunigtem Raum geht im neuen Jahrhundert auf molekularer Ebene weiter und gentherapeutische Ansätze sind bereits zu erkennen, wenn auch noch wenig konkret fassbar. Es ist zu hoffen, da unser Genpool evolutionsgeschichtlich aus dem „schwerelosen“ Meeresraum stammt, dass weitere Buchbände der Serie „Lung Biology in Health and Disease“ zum Thema „Microgravity“ mit vielen neuen Erkenntnissen folgen werden.

Heinrich Matthys, Freiburg 